Europhys. Lett., 13 (1), pp. 61-66 (1990)

\title{
The Most Simple Icosahedral Quasi-Crystal with Continuous Phasons.
}

\author{
M. WIRTH and H.-R. TREBIN \\ Institut für Theoretische und Angewandte Physik, Universität Stuttgart \\ Pfaffenwaldring 57, 7000 Stuttgart 80, F.R.G.
}

(received 20 April 1990; accepted in final form 28 June 1990)

PACS. 61.50E - Crystal symmetry; models and space groups, crystalline systems and classes. PACS. 61.90 - Other topics in structure of liquids and solids.

\begin{abstract}
An icosahedral quasi-crystal with continuous phason modes has been constructed in the form of five perfect intergrown FCC-lattices, with a finite distance between all atoms. Five is the minimal number possible. The model is essentially unique. The pair correlation function and the Fourier intensities are calculated. An analytic expression is presented for the displacement of the atoms under a phason shift.
\end{abstract}

\section{Introduction.}

Two major classes of incommensurate systems have been studied in the literature, namely the modulated crystals and the quasi-crystals. Both can be described as cuts of higher-dimensional periodic structures [1]. They differ significantly in the behaviour under a phason mode. When such a mode passes through a modulated crystal, as a rule the atoms move continuously, whereas in the classical quasi-crystal models [2,3] many atoms perform discontinuous jumps. Until recently it was believed that a continuous motion under a phason mode is not compatible with icosahedral symmetry [4]. But Kalugin and Levitov reported a counterexample [5], and soon afterwards Levitov constructed an infinite family of icosahedral quasi-crystals with continuous phason modes [6]. His model consists of 60 perfect periodic lattices related through the symmetry operations of the icosahedral group. He showed that it is possible to fulfil the hard-core condition, i.e. to maintain a finite distance between the atoms, even without adding a modulation. In this letter we show that among Levitov's models there is essentially one consisting of five lattices only and having maximal interatomic separation.

\section{The model.}

We denote by $\boldsymbol{Y}$ the proper $\left(^{1}\right)$ icosahedral group 532 of 60 elements. The minimal dimension for periodic systems which possess $\boldsymbol{Y}$ as point group is six. So we commence with

( $\left.{ }^{1}\right)$ No use is made of $Y_{\mathrm{I}}=\overline{5} \overline{3}(2 / \mathrm{m})$, which contains the inversion operation. As seems evident (and can be shown for our special model, but no general proof has been published yet) this symmetry does not permit structures with continuous phasons and hard cores. 
a.simple cubic lattice $\boldsymbol{G}^{6}$ in six-dimensional space $\boldsymbol{R}^{6}$, endow it with a basis of threedimensional atomic surfaces and cut it with a three-dimensional plane to arrive at the quasiperiodic three-dimensional structure [7]. The six-dimensional space group is supposed to be icosahedral and symmorphic (for a full classification of the icosahedral space groups in $\boldsymbol{R}^{6}$ see [8]).

Under the action of the symmetry elements $g \in \boldsymbol{Y}$ on $\boldsymbol{R}^{6}$, expressed by orthogonal $(6 \times 6)$ matrices $D_{g}^{6}, \boldsymbol{R}^{6}$ splits uniquely into two three-dimensional invariant subspaces $\boldsymbol{R}^{6}=$ $=\boldsymbol{E}^{\perp} \oplus \boldsymbol{E}^{\|}$, and the matrices $\boldsymbol{D}_{g}^{6}$ decompose into two $(3 \times 3)$-block matrices, $\boldsymbol{D}_{g}^{6}=\boldsymbol{D}_{g}^{\perp} \oplus \boldsymbol{D}_{g}^{\|}$. $\boldsymbol{D}_{g}^{\perp}$ and $\boldsymbol{D}_{g}^{\|}$are elements of two irreducible but inequivalent three-dimensional representations of $\boldsymbol{Y}$. Our cut plane is $\boldsymbol{E}^{\|}$which may be translated by a shift vector $\gamma^{\perp}$.

The atoms of the six-dimensional crystal are three-dimensional surfaces $\mathcal{S}$ transversal to the plane $\boldsymbol{E}^{\|}$(i.e. they always cut $\boldsymbol{E}^{\|}$under a finite angle). Owing to the condition of continuous phason modes, these surfaces are the graphs of continuous mappings $\boldsymbol{F}: \boldsymbol{E}^{\perp} \mapsto$ $\mapsto \boldsymbol{E}^{\|}, \mathcal{S}=\left\{\left(\boldsymbol{x}^{\perp}, \boldsymbol{x}^{\prime \prime}\right) \mid \boldsymbol{x}^{\prime \prime}=\boldsymbol{F}\left(\boldsymbol{x}^{\perp}\right)\right\}$. As is shown in [4] and can be simply visualized in $1+1$ dimensions, $\mathcal{S}$ has to be periodic over a three-dimensional lattice plane of $G^{6}$. In the simplest case $\mathcal{S}$ is flat (i.e. no modulation is present) and is parallel to the lattice plane. The corresponding mapping $\boldsymbol{F}$ then is affine: $\boldsymbol{F}\left(\boldsymbol{x}^{\perp}\right)=\boldsymbol{A}\left(\boldsymbol{x}^{\perp}\right)+\boldsymbol{a}^{\|}$, where $\boldsymbol{A}$ is linear and $\boldsymbol{a}^{\|}$is a constant shift. In this case $\mathcal{S}$ is denoted $\mathcal{S}_{A}$, and each point of the cut $\left(\boldsymbol{G}^{6}+\mathcal{S}_{A}\right) \cap\left(\boldsymbol{E}^{\|}+\gamma^{\perp}\right)$ can be expressed in obvious notation as

$$
\boldsymbol{n}^{\|}-\boldsymbol{A}\left(\boldsymbol{n}^{\perp}\right)+\boldsymbol{a}^{\|}+\boldsymbol{A}\left(\gamma^{\perp}\right), \quad \text { with } \boldsymbol{n} \in \boldsymbol{G}^{6}
$$

All these points together form a three-dimensional Bravais lattice.

When $\mathcal{S}_{A}$ is rotated by an element $g \in \boldsymbol{Y}$, or shifted by a six-dimensional lattice vector, the result also is an admissible atomic surface due to the icosahedral space group symmetry. So in general the quasi-crystal consists of 60 different interpenetrating lattices [5,6]. This number reduces, however, if $\mathcal{S}_{A}$ is left invariant as a whole by a subgroup of $\boldsymbol{Y}$ up to a shift vector out of the lattice $G^{6}$. The reduction is maximal, if this subgroup is a maximal one of $Y$, namely a tetrahedral group $\boldsymbol{T}$. As is well known, $\boldsymbol{Y}$ contains five such subgroups, and we will identify one of them with $T$.

A necessary condition for the invariance is that $\boldsymbol{A} \boldsymbol{D}_{g}^{\perp}=\boldsymbol{D}_{g}^{\|} \boldsymbol{A}$ for all $g \in \boldsymbol{T}$. For the special orthonormal bases of $\boldsymbol{E}^{\|}$and $\boldsymbol{E}^{\perp}$ introduced in [9], namely along the directions of twofold rotation axes of $D_{g}^{\|}$and $D_{g}^{\perp}$, respectively, we have $D_{g}^{\perp}=D_{g}^{\|}$for all $g \in T$. Since the matrices $\boldsymbol{D}_{g}^{\|}, g \in \boldsymbol{T}$ form an irreducible representation of $\boldsymbol{T}$, we conclude from Schur's lemma that $\boldsymbol{A}$ is a multiple of the unit matrix, i.e. $\boldsymbol{A}=\lambda_{A} 1$.

We start with $\mathcal{S}_{A}$ and act on it with the elements of the tetrahedral group $\boldsymbol{T}$. Since $\boldsymbol{T}$ is a group of order 12, in general we obtain 12 different atomic surfaces, which for the above choice of $A$ are all parallel. The three-dimensional cut yields 12 parallel cubic lattices. To reduce this number $\boldsymbol{a}^{\|}$has to be chosen on a symmetry axis of the tetrahedral group. The most symmetrical point is $\boldsymbol{a}^{\|}=0$, but conflicts with the hard-core condition. An explicit calculation of the separations of the atomic planes in six dimensions yields intersections, if we place $\boldsymbol{a}^{\|}$on a twofold axis of $T$, so this choice also has to be excluded. To arrive at a threedimensional structure with less than $5 \times 12$ parallel lattices, the only possible choice is a shift vector $\boldsymbol{a}^{\|}$situated on a threefold axis of $\boldsymbol{T}$. Without loss of generality we can set $\boldsymbol{a}^{\|}=\mu(1,1,1)$. Now groups of three lattices coincide and we are left with four. For full invariance under the icosahedral group, we operate successively with a group element $g_{5}$ of order five on our structure and arrive at five groups of four parallel cubic lattices. At this point we still have two parameters free: $\lambda_{A}$ and $\mu$.

A variation of $\mu$ only adjusts the relative positions of the lattices and determines the minimal interatomic distance. This shortest length in our crystal structure takes a maximum 
value for $\mu=(1 / 4) d$ ( $d=$ lattice constant of one of the lattices), namely $d_{\min }=$ $=(1 /(2 \sqrt{3})) d \approx 0.289 d$. For this special value of $\mu$ the four parallel cubic lattices merge into one FCC-lattice $G_{0}^{\|}$. Now we have obtained our minimal structure of five intergrown FCClattices:

$$
G_{\alpha}^{\|}=D_{g_{5}}^{\|} G_{0}^{\|}, \quad \alpha=0, \ldots, 4
$$

If henceforth we talk about the lattice constant or the Miller indices of an FCC- and BCClattice we refer to the standard nonprimitive orthogonal lattice basis. For the value of $a^{\prime \prime}$ chosen, variations $\left(^{2}\right)$ in $\lambda_{A}$ merely rescale the three-dimensional structure, so the model is essentially unique.

A further reduction of the number of lattices would only be possible if $\mathcal{S}_{A}$ were invariant under the complete icosahedral point group $\boldsymbol{Y}\left(\bmod G^{6}\right)$. But such a situation would lead to a dense point set in three-dimensional space, since then $\mathcal{S}_{A}$ is forced to be parallel to $\boldsymbol{E}^{\perp}$.

Thus the requirement that the number of lattices is minimal and that the minimal interatomic distance is maximal singles out one model of the countable infinite family constructed by Levitov [6].

\section{Properties.}

This simple model allows to express explicitly some important physical properties such as the pair correlation function or the structure factor.

3.1. Pair correlation function. - The formal definition of the pair correlation function reads

$$
\boldsymbol{g}(\boldsymbol{x})=\lim _{V \rightarrow \infty} \frac{1}{V} \int_{V} \rho(\boldsymbol{x}-\boldsymbol{y}) \rho(\boldsymbol{y}) \mathrm{d}^{3} \boldsymbol{y}
$$

where $\rho$ is the mass density of the structure under consideration. For a monoatomic crystal it is proportional to the density of the difference vectors between arbitrary pairs of atoms in the crystal. For our structure $\boldsymbol{g}$ is the sum of two parts: $\boldsymbol{g}=\boldsymbol{g}_{\text {disc }}+\boldsymbol{g}_{\text {cont }}$.

$\boldsymbol{g}_{\text {disc }}$ is the density of interatomic distance vectors between two atoms that belong both to the same lattice, and as such is a superposition of the pair correlation functions for the five FCC-lattices (2):

$$
g_{\text {disc }}:=\frac{1}{5} \sum_{\alpha=0}^{4} \delta_{G_{\alpha}^{\prime \prime}}
$$

where for every point set $\boldsymbol{U}$ we defined

$$
\delta_{U}(x):=\sum_{x_{0} \in U} \delta\left(x-x_{0}\right)
$$

$\boldsymbol{g}_{\text {cont }}$ is the distribution of the distance vectors between atoms that belong to different

${ }^{2}$ ) Since $\boldsymbol{A}$ has to describe a lattice plane, not all values of $\lambda_{A}$ are allowed. But it can be shown that the permissible values are dense in $\boldsymbol{R}$. 
lattices $G_{\alpha}^{\|}$. It is not discrete, rather is equally distributed over a decagrid, whose twodimensional planes are parallel to the faces of an icosahedron. The points $\boldsymbol{x}^{\prime \prime}$ of the grid planes are described by the Hesse-form

$$
\left\langle\boldsymbol{x}^{\|} \mid \boldsymbol{e}\right\rangle=\left(\frac{1}{2}+m\right) \frac{d}{\sqrt{3}},
$$

where the unit vector $\boldsymbol{e}$ points in the direction of a threefold axis of $\boldsymbol{D}_{g}^{\prime \prime}, g \in \boldsymbol{Y}$, and $m$ is an integer. $\boldsymbol{g}_{\text {cont }}$ then may be written

$$
\boldsymbol{g}_{\text {cont }}:=\frac{8}{5 \sqrt{3} d^{2}} \delta_{\text {grid }} .
$$

3.2. Radial distribution function. - The radial distribution function $\rho$ is formally related to the pair correlation function through the equation

$$
\int_{0}^{R} \rho(r) r^{2} \mathrm{~d} r=\int_{\|x\| \leqslant R} \boldsymbol{g}(\boldsymbol{x}) \mathrm{d}^{3} \boldsymbol{x}
$$

and also consists of two parts:

$\rho_{\text {dise }}$ is the radial distribution function of an FCC-lattice;

Pcont is a continuous part, resulting from pairs of atoms which belong to different lattices $G_{\alpha}^{\|}$. It is found by spherical averaging over the grid structure of $\boldsymbol{g}_{\text {cont }}$ :

$$
\rho_{\text {cont }}(r)=\frac{64}{\sqrt{3} d^{2}} \frac{\operatorname{ceil}\left(\left(r-r_{0}\right) / 2 r_{0}\right)}{r} ; \quad r_{0}=\frac{1}{2 \sqrt{3}} d
$$

(ceil $(r)$ is the smallest integer that is greater than or equal to $r$.)

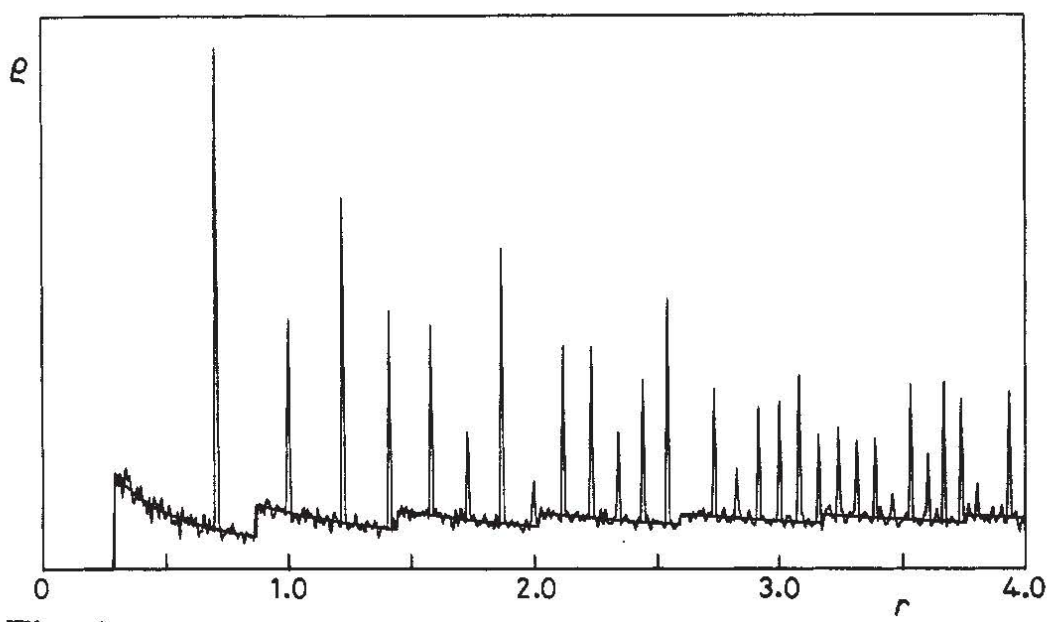

Fig. 1.

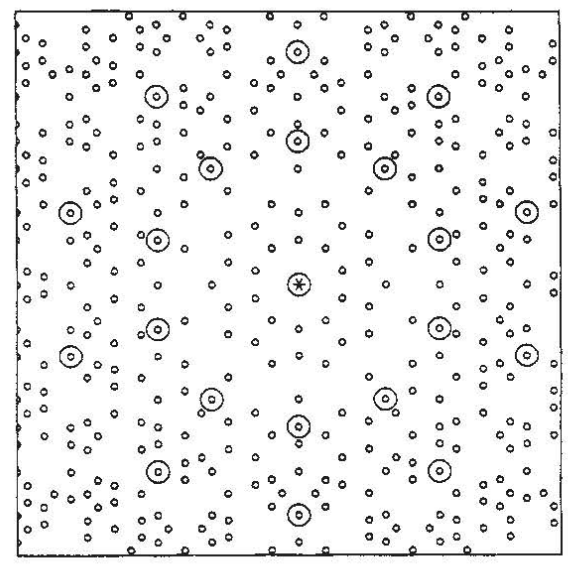

Fig. 2.

Fig. 1. - Radial distribution function. The unit length is the FCC-lattice constant $d$. The noisy curve comes from a finite computer model (20000 atoms), whereas the smooth curve is obtained analytically. The discrete part is exactly equal to that of a single FCC-lattice.

Fig. 2. - Projection of the scattering vectors onto a plane perpendicular to a fivefold axis. There are two intensities, the standard one (small circles) and a fourfold one (large circles). 
The startling presence of a continuous background in the radial distribution function is a generic feature of modulated structures with two or more differently oriented atomic surfaces $\mathcal{S}$ (under the assumption that the cut plane forms irrational angles with the higherdimensional lattice). Through modulation this continuous part may be lowered, but cannot be avoided completely without tearing the surface apart.

3.3. Intensities in reciprocal space. - In contrast to the continuous part of the pair correlation function, its Fourier transform, denoted $\hat{g}$, is purely discrete. In a first approximation $g$ is proportional to the intensities of X-ray diffraction experiments. Peaks occur only at points belonging to the BCC-lattices $G_{\alpha}^{11 *}$ which are reciprocal to the FCClattices $G_{\alpha}^{\|}$. Due to intersections of the reciprocal lattices $G_{\alpha}^{\| *}$, extinctions and amplifications of peaks occur on the threefold axes of $D_{g}^{\|}$. Peaks with odd indices on diagonals of $G_{a}^{\| *}$ are not present, whereas even indexed peaks have the fourfold intensity.

The peak positions of this structure factor do not match any of the presently observed diffraction patterns. In particular our scheme does not contain the golden number $\tau$ in the shell radii, as is the case for all the measured icosahedral alloys.

3.4. Structure factor. - Since the structure factor is essentially the spherical average of the Fourier intensity, it is clear that it also is purely discrete, and that the peak positions are those of an FCC-lattice. Only the intensities of single peaks are altered. To be more precise, peaks with three equal even Miller indices are stronger and those with three equal odd indices are damped or absent.

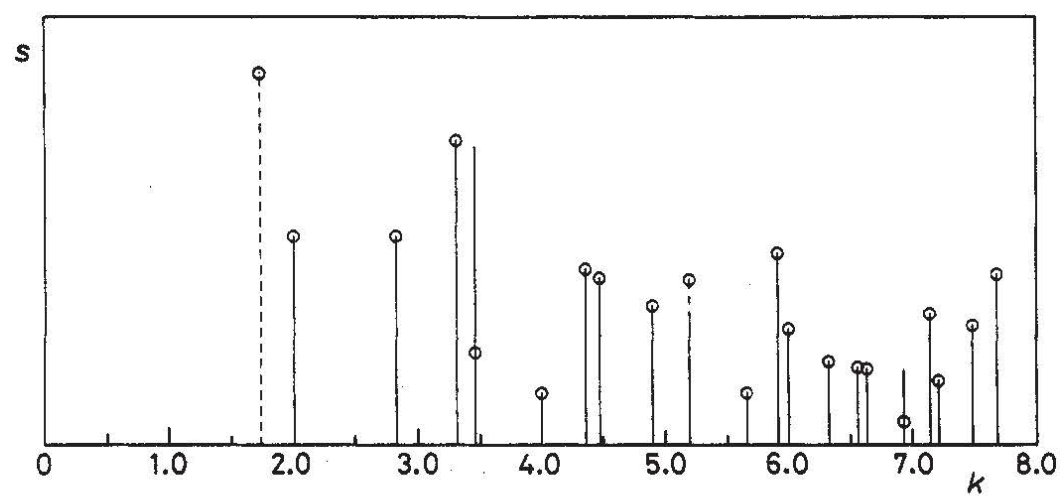

Fig. 3. - Structure factor. The solid lines mark the intensities of our five-lattice model, whereas the dashed lines topped with a circle indicate the peak positions of a single FCC-lattice (scaled by 5 ). The unit is the reciprocal lattice constant $2 \pi / d$.

\section{Phasons.}

If a phason passes through a modulated structure the points of the superspace $\left(\boldsymbol{R}^{6}\right.$ in our case) are shifted perpendicular to the physical space $\boldsymbol{E}^{\|}$. Under this deformation the atomic surfaces are bent, and their intersections with $\boldsymbol{E}^{\|}$move. This motion may be described as follows. Let $\Phi:\left(x^{\perp}, x^{\prime \prime}\right) \mapsto\left(x^{\perp}+m\left(x^{\prime \prime}\right), x^{\prime \prime}\right)$ be a phasonic deformation of $\boldsymbol{R}^{6}$. If $\boldsymbol{x}_{0}^{\|}$is a point resulting from the cut of $\boldsymbol{E}^{\prime \prime}$ with an atomic surface $\mathcal{J}_{\boldsymbol{A}}$, the new position $\boldsymbol{x}_{m}^{\prime \prime}$ after deformation is given by the equation: $(1+\boldsymbol{A} \boldsymbol{m})\left(\boldsymbol{x}_{m}^{\| \prime}\right)=\boldsymbol{x}_{0}^{\prime \prime}$. So a phason corresponds to a different phonon in each of the five lattices.

The analytical inversion of the mapping $1+\boldsymbol{A m}$ is rarely possible, but if $\boldsymbol{m}$ is slowly 
varying (compared to the amplitude of the displacement) one can use a linear approximation:

$$
x_{m}^{\| \prime}=x_{0}^{\|}-\left(A \nabla m\left(x_{0}^{\|}\right)+1\right)^{-1}\left(A m\left(x_{0}^{\|}\right)\right)
$$

If for example $\boldsymbol{m}$ is a shift in only one direction: $\boldsymbol{m}\left(\boldsymbol{x}^{\prime \prime}\right)=f\left(\boldsymbol{x}^{\prime \prime}\right) \boldsymbol{b}^{\perp}, f\left(\boldsymbol{x}^{\prime \prime}\right) \in \boldsymbol{R} ; \boldsymbol{b}^{\perp} \in \boldsymbol{E}^{\perp}$, the inversion of (5) is possible:

$$
\boldsymbol{x}_{m}^{\|}=\boldsymbol{x}_{0}^{\|}-\frac{f\left(\boldsymbol{x}_{0}^{\|}\right)}{1+\left\langle\nabla f\left(\boldsymbol{x}_{0}^{\| \prime}\right) \mid \boldsymbol{A}\left(\boldsymbol{b}^{\perp}\right)\right\rangle} \boldsymbol{A}\left(\boldsymbol{b}^{\perp}\right) .
$$

\section{Summary and remarks.}

In this letter we have presented an icosahedral quasi-crystalline structure with the following properties: it consists of five perfect, interpenetrating FCC-lattices, yet there is a minimal separation of $0.289 d$ of the atoms, where $d$ is the FCC-lattice constant. The radial distribution function has the same peaks as for a single FCC-lattice, but with a continuous background. Also the peak positions of the structure factor are those as for a FCC-lattice. The Fourier transform of the pair correlation function, which is observed in diffraction experiments, yields a Bragg pattern with icosahedral symmetry, but is perfectly discrete, in contrast to the standard three-dimensional Penrose pattern, whose diffraction pattern is dense in reciprocal space.

So far we considered structures with a SC-lattice and a symmorphic space group in $\boldsymbol{R}^{6}$ only. But the other icosahedral space groups may be considered as subgroups of this one, and hence it is possible to construct five lattice models for all six-dimensional icosahedral space groups, which will be reported elsewhere.

\section{REFERENCES}

[1] Janssen T. and Janner A., Adv. Phys., 36 (1987) 519.

[2] Penrose R., Bull. Instrum. Math. Appl., 10 (1974) 266.

[3] Kramer P. and Neri R., Acta Crystallogr. Sect. A, 40 (1985) 580.

[4] Frenkel D. M., Henley C. L. and Siggia E. D., Phys. Rev. B, 34 (1986) 3649.

[5] Kalugin P. A. and Levitov L. S., Int. J. Mod. Phys. B, 3 (1989) 877.

[6] Levitov L. S., J. Phys. (Paris), 49 (1988) 1835.

[7] BaK P., Phys. Rev. Lett., 56 (1986) 861.

[8] Levitov L. S. and Rhyner J., J. Phys. (Paris), 49 (1988) 1835.

[9] Cahn J. W., Shechtman D. and Gratias D., J. Mater. Res., 1 (1986) 13. 